\title{
Finger Vein Recognition based on Matching Score-Level Fusion of Gabor Features
}

\author{
Yu Lu*, Sook Yoon, Dong Sun Park
}

\begin{abstract}
Most methods for fusion-based finger vein recognition were to fuse different features or matching scores from more than one trait to improve performance. To overcome the shortcomings of "the curse of dimensionality" and additional running time in feature extraction, in this paper, we propose a finger vein recognition technology based on matching score-level fusion of a single trait. To enhance the quality of finger vein image, the contrast-limited adaptive histogram equalization (CLAHE) method is utilized and it improves the local contrast of normalized image after ROI detection. Gabor features are then extracted from eight channels based on a bank of Gabor filters. Instead of using the features for the recognition directly, we analyze the contributions of Gabor feature from each channel and apply a weighted matching score-level fusion rule to get the final matching score, which will be used for the last recognition. Experimental results demonstrate the CLAHE method is effective to enhance the finger vein image quality and the proposed matching score-level fusion shows better recognition performance.
\end{abstract}

Key Words : Biometrics, Finger vein recognition, Matching score-level fusion, Gabor filter, Contrast-limited adaptive histogram equalization

\section{I . Introduction}

Personal identification using biometric features has been on a rapid development over the last two decades. Traditional biometric methods which utilize features such as the fingerprints, palmprint, iris, facial features, and hand shape to identify individuals provide effective approaches for many real applications. However, no biometric has been proved to be perfectly reliable, robust and secure. For example, palmprint and fingerprint are susceptible to forgery since they are exposed outside the human body. Face recognition may easily be affected by illumination and occlusion and iris recognition is also considered as unfriendly for its unpleasant data acquisition $\operatorname{process}^{[1]}$.

Recently, a new biometric technology based on human finger vein patterns has attracted the attentions of biometrics-based identification research community ${ }^{[2]}$. Compared with the traditional biometric features, finger vein recognition has the following advantages ${ }^{[3]}$ : 1) immunity to forgery, since finger veins are hidden underneath the skin's surface as a part of internal physiological characteristics, 2) friendliness, whereas a near-infrared light (wavelengths between 700 and 1000 nanometers) is used for contactless, non-invasive imaging that ensures both convenience and cleanliness for the user, and 3) polymorphism, which has been proved that each finger from the same person has unique vein

※ This work is supported by the State Scholarship Fund organized by the China Scholarship Council.

※ This work is supported by the Ministry of Education, Science Technology (MEST) and National Research Foundation of Korea (NRF) through the Human Resource Training Project for Regional Innovation.

- 주저자 : 전북대학교 대학원 전자정보공학부 전자공학전공, luyu0311@gmail.com, 학생회원

○교신저자 : 목포대학교 멀티미디어공학과, syoon@mokpo.ac.kr, 정회원

* 전북대학교 전자공학부, IT융합연구센터, dspark@jbnu.ac.kr, 종신회원

논문번호 : KICS2012-11-530, 접수일자 : 2012년 11월 13일, 최종논문접수일자 : 2013년 2월 4일 
$\operatorname{pattern}^{[4]}$.

As the properties described above, personal authentication using finger vein images has received certain development over the last decade. Miura et al. and Zhang et al. proposed a finger vein extraction method based on repeated line tracking $^{[5]}$ and curvelet information of the image profile with a locally interconnected neural network $^{[6]}$, respectively. Principal component analysis and the neural network technique are also used for finger-vein pattern identification by $\mathrm{Wu}$ et al. ${ }^{[7]}$ To further improve the performance of finger vein recognition system, some approaches use the fused features for identification. Lee et al. proposed to extract binary patterns of finger images including multimodal feature of veins and finger geometries for recognition ${ }^{[8]}$. Yang et al. proposed a novel finger-vein recognition method based on the feature level fusion of global and local feature ${ }^{[9]}$. Guan et al. proposed to fuse the features from wavelet moment and horizontal and vertical 2DPCA for the recognition ${ }^{[10]}$. These feature-level fusion methods commonly improve the matching accuracy, but they tend to increase the computational complexity of authentication systems.

In this paper, we proposed a finger vein recognition method using matching score-level fusion from a single set of simple features to lower the computational complexity while to slightly increase the matching accuracy. The flow chart of the proposed method is shown in Fig 1. The Region of Interest (ROI) is detected first. Then, adaptive wavelet shrinkage (AWS) denoising method and Contrast-Limited Adaptive Histogram Equalization (CLAHE) method are respectively used for image denoising and image enhancement. The Gabor features from 8 channels based on a bank of Gabor filters are extracted to obtain the 8 matching scores. Finally, based on the weight-fused matching score, classification using nearest neighbor classifier with cosine distance measure is performed. The contribution of this paper is that a weighted sum rule is used for matching scores fusion from one trait.

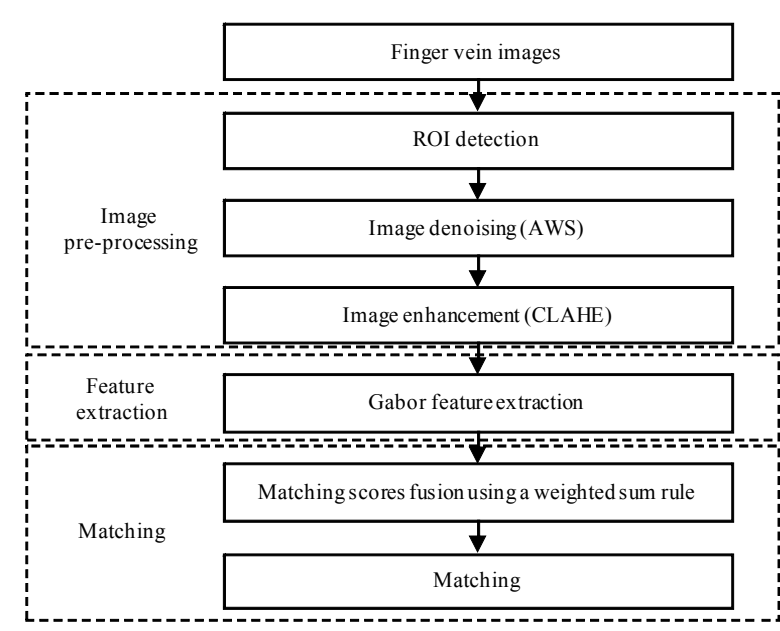

Fig. 1. Flow chart of the proposed method

\section{Image Preprocessing}

Owing to some kinds of noise or strong reflection resulted from the skin's surface and shallow penetration of light under the skin, the original finger vein image contrast is small which makes it not distinct enough for identification ${ }^{[11]}$. The typical filter-based image enhancement methods are adopted for poor quality finger vein images to improve their contrast ${ }^{[12][13]}$. Wang et al. enhanced the region of interest of finger vein images using Genetic Programming (GP) algorithm $^{[14]}$. Yang et al. solved the problem of finger-vein image degradation by removing the scattering in finger vein images ${ }^{[15]}$.

Image preprocessing in this section includes the following three processes: ROI detection, image denoising and image enhancement. The procedure of these three processes is demonstrated in Fig. 2.

\subsection{ROI Detection}

As shown in Fig. 2(a), since the background is not well handled, a finger contour (Fig. 2(b)) is obtained with 'open' operation. Then, Hough transform is used to detect two finger edges in the contour image, which is unaffected by image noise. Two boundaries of these fingers are well displayed in Fig. 2(c) with white color. To decide an ROI, a region with fixed geometrical position and size is chosen to generate a normalized ROI (the white region in Fig. 2(c)). 


\subsection{Image Denoising}

Wavelet transform based image denoising techniques have been proved to be effective with their compressibility of information signal and incompressibility of noise signal. An adaptive wavelet shrinkage (AWS) denoising method ${ }^{[16]}$ is used in the image denoising process. It uses wavelet transform to extract information on sharp variation in multi-resolution images and applies shrinkage function which adapts image features. It has the merits of low complexity and good performance. Fig. 2(e) shows a finger vein image after image denoising using the adaptive wavelet shrinkage.

\subsection{Image Enhancement}

Since finger vein images are not always with high quality due to the varying tissues and bones, and even illuminations, efficient image enhancement method is necessary to improve the image quality. For image enhancement, we utilized the Contrast-limited Adaptive Histogram Equalization (CLAHE). It differs from the ordinary histogram equalization in the respect that computes several histograms, each corresponding to a distinct section of an image, and uses them to redistribute the lightness values of the image. It is therefore suitable for improving local contrast of an image and bringing out more in detail. Moreover, CLAHE can prevent overamplification of noise that AHE can give rise to ${ }^{[17]}$. Fig. 2(f) shows much clearer and more details than the original ROI (Fig. 2(d)).

\section{Gabor Feature Extraction}

Gabor filter is very useful in pattern recognition applications such as iris recognition ${ }^{[18]}$, fingerprint recognition ${ }^{[19]}$ and hand vein recognition ${ }^{[20]}$, which is powerful in capturing some specific texture characteristics in images. As even-symmetric Gabor filter is suitable for ridge detection for an image, a filter bank consisting of even-symmetric Gabor filters with one scale and 8 rotations is defined as follow:

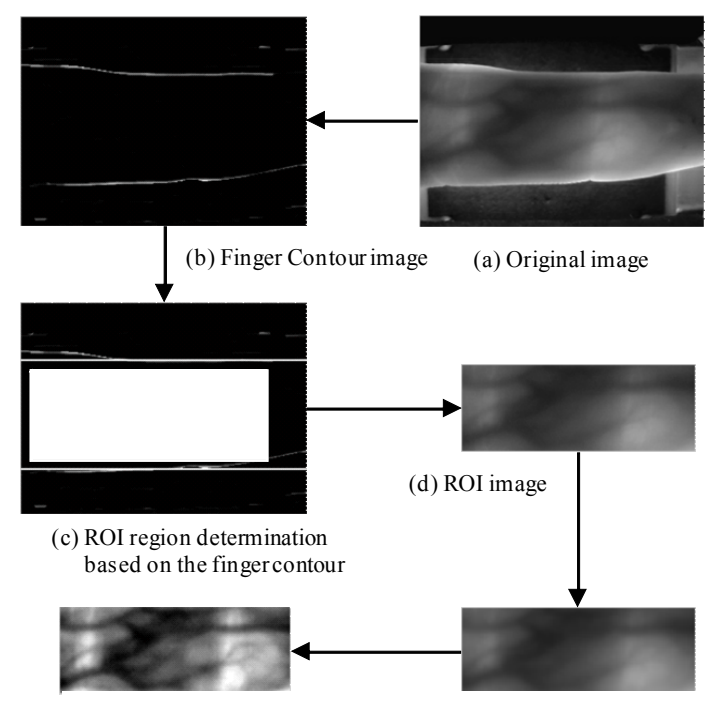

(f) Enhanced image

(e) Image after denoising

Fig. 2. ROI detection, image denoising, and image enhancement for finger vein image

$$
\begin{aligned}
& G_{k}^{R}\left(x, y, \gamma, \sigma, f_{k}, \theta_{k}\right)= \\
& \quad \rho \exp \left\{-\frac{1}{2}\left(\frac{x_{\theta k}^{2}+\gamma^{2} y_{\theta k}^{2}}{\sigma^{2}}\right)\right\} \cos \left(2 \pi f_{k} x_{\theta k}\right)
\end{aligned}
$$

where $\gamma$ and $\sigma$ denote an aspect ratio of the elliptical Gaussian envelope and a standard deviation. $\rho=\frac{\gamma}{2 \pi \sigma^{2}}$ is a scale index, $k=1,2, \ldots, 8$ is a channel index, $\theta_{k}=\frac{k \pi}{8}$ and $f_{k}$ represent an orientation and a center frequency of an even-symmetric Gabor filter at the $k^{\text {th }}$ channel respectively. $x_{\theta k}$ and $y_{\theta k}$ are rotated versions of coordinates $(x, y)$ of a Gabor filter, $\hat{j} \equiv \sqrt{-1}$.

Suppose $I(x, y)$ is a normalized finger vein image, its corresponding filtered image $F_{\gamma, \sigma, f_{k}, \theta_{k}}(x, y)$ is generated by the following formula:

$$
\begin{aligned}
& F_{\gamma, \sigma, f_{k}, \theta_{k}}(x, y)= \\
& \quad G_{k}^{R}\left(x, y, \gamma, \sigma, f_{k}, \theta_{k}\right) \star I(x, y)
\end{aligned}
$$

in which $\star$ is a convolution operation. Using a bank of Gabor filters, 8 filtered images are obtained, which are shown in Fig. 3(a). 


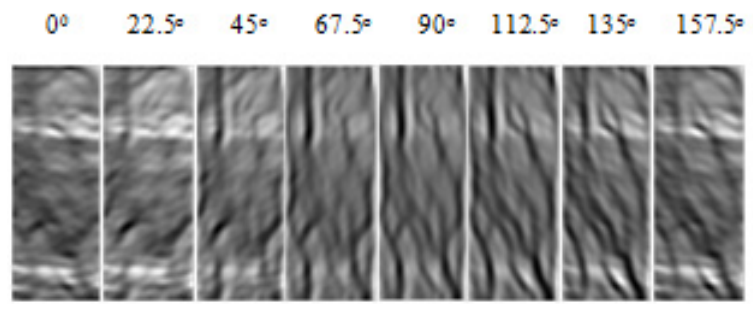

(a) Output of a bank of even-symmetric Gabor filters in one scale

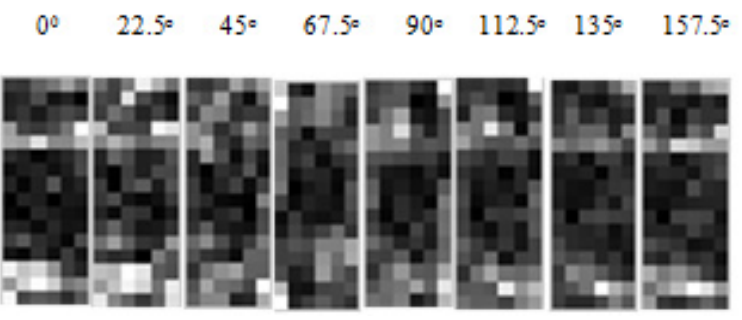

(b) Corresponding AAD images

Fig.3. Output of a filter bank of even-symmetric Gabor filters with one scale and its corresponding AAD images.

To obtain discriminative local features, an average absolute deviation (AAD) in $16 \times 6$ blocks of each filtered image is constructed ${ }^{[2][21]}$. Thus, for a normalized finger vein image, a feature vector with $768(16 \times 6 \times 8) \quad$ AAD values can be extracted from 768 blocks at one scale.

\section{Matching Score-level Fusion}

Multiple biometrics is an effective way to improve a performance of recognition system, which includes different sources ${ }^{[22]}$, whereas these sources may come from (a) multiple biometrics traits, such as iris and palmprint ${ }^{[23]}$, finger vein, fingerprint and face ${ }^{[24]}$ (and so on), or (b) multiple sensors, multiple instances, multiple snapshots, or multiple matching algorithms of a single biometric trait. Yang et al. fused global features (AAD) and local features (Moment Invariant features) for finger vein recognition ${ }^{[9]}$. Park et al. studied on a finger vein recognition by combining global and local features based on $\mathrm{SVM}^{[25]}$. However, although the fused feature vector has richer source of information, features from these modalities may not be compatible.
Furthermore, large dimensionality of the fused feature space may bring irrelevant and the redundant information.

Matching score-level fusion which combines the matching scores from the individual matchers focuses on different integration approaches. Matching score fusion techniques can be divided into three categories as set forth ${ }^{[21]}$ : (a) density-based matching score fusion; (b) classifier-based matching score fusion; and (c) combination-based or transformation-based matching score fusion. We used (c) method among the list of three methods categorized above. With the combination-based method, product rule, sum rule, max rule and min rule are often used to determine the final score. In this paper, a weighted sum rule is proposed to fuse the matching scores from the AAD features of 8 channels.

Suppose $A A D_{k} \mathrm{~s}$ are the average absolute deviation features obtained from a bank of Gabor filters, and $k=1,2, \ldots, 8$. The corresponding Recognition Rates(RR) $R_{k} \mathrm{~s}$ are obtained from $A A D_{k} \mathrm{~s}$ using the nearest neighbour classifier with cosine distance matching and used as Matching scores $S_{k} \mathrm{~s}$ corresponding to $A A D_{k} \mathrm{~s}$. To highlight the contributions of AAD features from different channels, a weighted sum rule is utilized to generate the final matching score used for the last identification. In this paper, $R_{k} \mathrm{~s}$ are firstly ranked in ascending order and one of serial numbers $\{1$, $2, \ldots, 8\}, n_{k}$, is assigned to $R_{k}$ according to its ranking of ascending order. And then $n_{k}$ is used to determine the weight, $w_{k}$, of each matching score $S_{k}$ using eq. (4). $w_{k}$ s are the corresponding weights of $\mathrm{AAD}$ features from 8 directions and the final matching score is defined as:

$$
\begin{aligned}
& S_{\text {final }}=w_{1} S_{1}+w_{2} S_{2}+\ldots+w_{8} S_{8} \\
& w_{k}=\frac{n_{k}}{1+2+\ldots+8}
\end{aligned}
$$

where $w_{1}+w_{2}+\ldots+w_{8}=1$. Hence, the higher recognition rate of $A A D_{k}$ from one direction, the 
larger weight will be endowed to this direction. Meanwhile, this direction contributes more to the last recognition and verification.

\section{Experimental results}

\subsection{Finger Vein Database}

The database used in this paper is from the Group of Machine Learning and Applications, Shandong University ${ }^{[1]}$. The database named SDUMLA-HMT is the Homologous Multi-modal Traits Database which includes real multimodal data from individuals.

To best of their knowledge, the finger vein database in SDUMLA-HMT is the first open finger vein database ${ }^{[26]}$. The device used to capture finger vein images is designed by Joint $\mathrm{Lab}$ for Intelligent Computing and Intelligent Systems of Wuhan University. In the capturing process, each person was asked to provide images for his/her index finger, middle finger and ring finger of both hands, and a collection for each of the 6 fingers is repeated for 6 times to obtain 36 finger vein images. Therefore, the finger vein database is composed of 3,816 images $320 \times 240$ in pixels. The geometrical size of ROI is $96 \times 256$. In our experiments, each finger is considered as one subject.

\subsection{Experimental Results}

Our experimental results includes image enhancement, identification and verification which are presented below.

\subsubsection{Image Enhancement}

Fig. 4 shows finger vein images using different image enhancement methods. As the vein randomly locates in a finger, the image enhancement methods in global way cannot enhance the contrast of image well enough. Unlike the global enhancement methods, contrast-limited adaptive histogram equalization (CLAHE) operates on small data region rather than an entire image. Each region's contrast is enhanced so that the histogram of each output region approximately matches the specified histogram. Meanwhile, CLAHE method can be limited in order to avoid amplifying the noise which might be present in the image. Therefore, the finger vein images enhanced by CLAHE show much clearer details than other images enhancement methods.

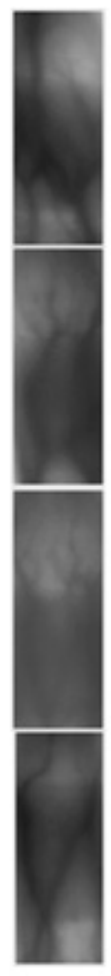

(a)
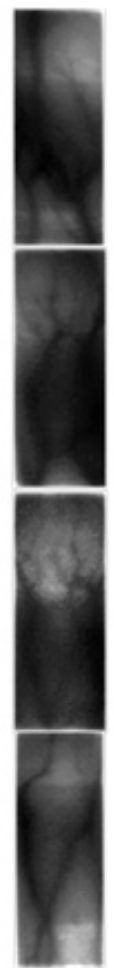

(b)

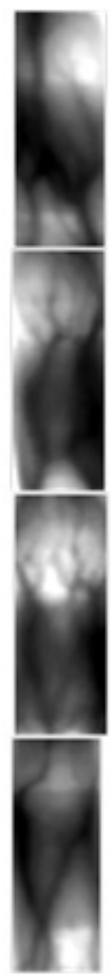

(c)

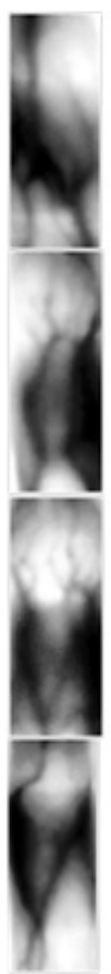

(d)

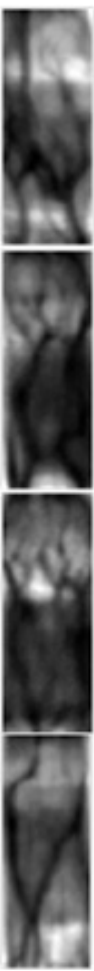

(f)
Fig.4. Comparisons of different image enhancement methods. (a) Original normalized image; (b) Gaussian high-pass filter; (c) Histogram modification; (d) Histogram equalization; (e) CLAHE

\subsubsection{Identification Experiments}

In these experiments, 3804 images from 634 fingers are used for recognition. We select three finger vein images from one individual as a training set and the rest as a test set. To verify the contribution from eight directions, recognition rates of $\mathrm{AAD}$ feature from eight directions are gained by nearest neighbor classifier with similarity measure of cosine distance. The cosine similarity (CS) between two vectors $x$ and $y$ is defined as ${ }^{[27]}$ :

$$
C S(x, y)=\frac{x \cdot y}{\|x\|\|y\|}
$$


The cosine similarity between the feature vector from training set and test set can be computed as follow:

$$
\begin{aligned}
& C S\left(\operatorname{Train}^{j}, \operatorname{Test}^{j}\right)=\frac{\operatorname{Train}^{j} \cdot \text { Test }^{j}}{\left\|\operatorname{Train}^{j}\right\| \| \text { Test }^{j} \|} \\
& =\frac{\sum_{i=1}^{n} \operatorname{Train}_{i}^{j} \times \operatorname{Test}_{i}^{j}}{\sqrt{\sum_{i=1}^{n}\left(\operatorname{Train}_{i}^{j}\right)^{2}} \sqrt{\sum_{i=1}^{n}\left(\operatorname{Test}_{i}^{j}\right)^{2}}}
\end{aligned}
$$

where $j=1,2, \ldots, 1902, i=1,2, \ldots, 96, \quad \operatorname{Train}^{j}$ and Test $^{j}$ are the AAD features from one training image and one test image respectively.

Fig. 5 shows performances using AAD features from different channels. The performance using all AAD features from 8 channels is $98.48 \%$, which is better than those merely using one channel's AAD feature. This is the reason that all 8 channels AAD features include abundant features from each direction. It should be emphasized that features from different channels have different performances which imply they should have different contributions when their matching scores are combined. For example, performance obtained by using one feature from the eighth direction is the best while a recognition rate using one feature from the fifth direction is the worst in all 8 channels. This means that the matching score from the eighth direction has the largest weight while the score from the fifth direction have the least weight.

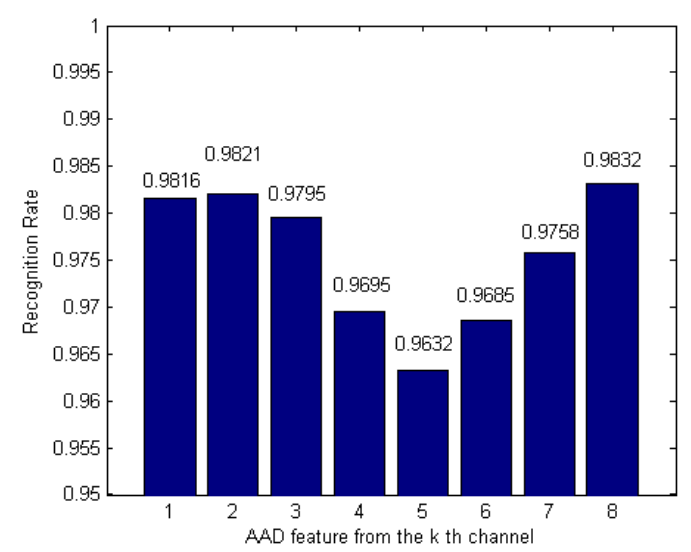

Fig.5. Recognition Rates using AAD features from 8 different channels
For matching scores from 8 channels, sum rule, $\max$ rule and min rule are often used to determine the final score. Following up our discussion, to emphasize the feature from different channels with different recognition contributions, a weighted sum rule is utilized for all the experiments in this paper. The weighted sum rule to obtain the last matching score is demonstrated as follow:

$$
\begin{gathered}
S_{\text {final }}=\frac{6}{36} S_{1}+\frac{7}{36} S_{2}+\frac{5}{36} S_{3}+\frac{3}{36} S_{4}+ \\
\frac{1}{36} S_{5}+\frac{2}{36} S_{6}+\frac{4}{36} S_{7}+\frac{8}{36} S_{8}
\end{gathered}
$$

Table 1 suggests the results generated by our fusion rule and other ordinary rules. As shown in the table, we can see that our method can obtain better performance on recognition rate than those using other common rules.

Meanwhile, Table 2 shows the comparisons of recognition rates of methods for the finger vein recognition. The pixel length step in the moment invariant method is 20. Thus, the feature vector of one finger image is $M I_{10 \times 7}$. The Gabor parameters for the feature-level fusion method are similar as those used in this paper, while the weights about global feature and local feature equal to those in [9]. Our matching-level fusion method displays better performances than other methods.

Table 1. Recognition rates with different fusion rules

\begin{tabular}{|c|c|}
\hline Fusion rules & Recognition Rate (\%) \\
\hline Sum rule & $98.63 \%$ \\
\hline Max rule & $98.37 \%$ \\
\hline Min rule & $97.79 \%$ \\
\hline Proposed rule & $98.79 \%$ \\
\hline
\end{tabular}

Table 2. Recognition rates with different methods

\begin{tabular}{|c|c|}
\hline Methods & Recognition Rate (\%) \\
\hline PCA $^{[28]}$ & $96.48 \%$ \\
\hline Moment Invariant $^{[29]}$ & $91.11 \%$ \\
\hline Feature-level Fusion $^{[9]}$ & $95.11 \%$ \\
\hline Proposed method $^{2}$ & $98.79 \%$ \\
\hline
\end{tabular}




\subsubsection{Verification Experiments}

In this experimental, we also select three finger vein images from one individual as training set while the left three images as test set. To evaluate the performance of this experiment, False Accept Rate (FAR) and False Rejection Rate (FRR) are reported. The FAR and FRR will change with the variation of threshold. The Equal Error Rate (EER) of a system is the value when FAR equals to FRR. Table 3 shows the EER using our method is smaller than those using other feature extraction methods, which demonstrates the proposed technology has better performance.

Table 3. Equal error rates with different methods

\begin{tabular}{|c|c|}
\hline Methods & Equal Error Rate (\%) \\
\hline PCA $^{[28]}$ & $5.84 \%$ \\
\hline Moment Invariant $^{[29]}$ & $9.15 \%$ \\
\hline Feature-level Fusion $^{[9]}$ & $5.38 \%$ \\
\hline Proposed method & $2.84 \%$ \\
\hline
\end{tabular}

Receiver Operating Characteristic (ROC) describes various pairs of FAR and FRR as threshold changes. Fig. 6 shows the ROC curves obtained by using different feature extraction methods. MI means the moment invariant method; FF is the feature-level based fusion method. The data result clearly shows the matching score-level fused method obtains better performance.

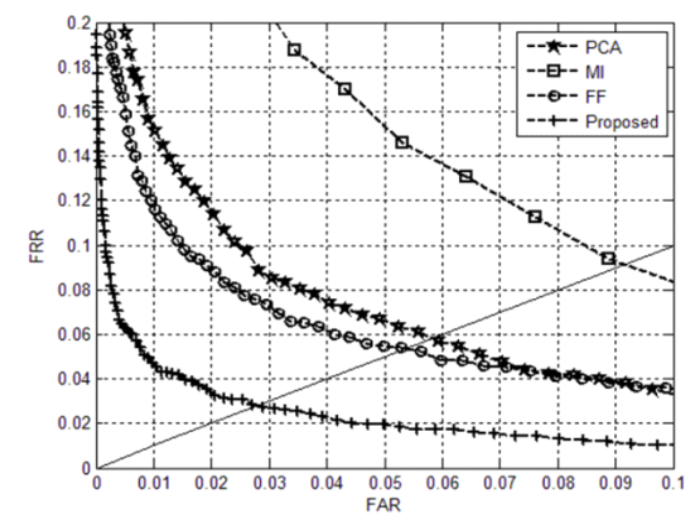

Fig. 6. ROC curves of different finger vein matching methods

\section{CONCLUSION}

Traditional fusion based finger vein recognition methods have the shortcomings of "the curse of dimensionality" and increased running time in extracting multiple features. To overcome the drawbacks, in this paper, we proposed a finger vein recognition method based on matching score-level fusion of Gabor features. In image preprocessing, multi-resolution method and contrast-limited adaptive histogram equalization method are applied for image denoising and enhancement. In the recognition and verification process, we discuss the contributions from 8 channels by using AAD features and present a weighted sum rule to fuse the matching scores from 8 channels. Our proposed method can obtain a recognition rate of $98.79 \%$ with the identification model and EER of $2.84 \%$ with the verification model. The experimental results demonstrate that the finger vein image quality is effectively enhanced and the proposed method is robust for personal authentication.

\section{References}

[1] C. Choi, B. Jun, "Robust-to-rotation Iris Recognition Using Local Gradient Orientation Histogram," The Journal of Korean Information and Communications Scociety, vol. 34, no. 3, pp. 268 - 273, March 2009.

[2] J F. Yang, Y S. Shi, J L, Yang, "Person identification based on finger-vein features," Computers in Human Behavior. vol. 28, no. 5, pp.1565-1570, Sep. 2011.

[3] J C. Hashimoto, "Finger vein authentication technology and its future," in Symposium on VLSI Circuits Digest of Technical Papers, pp. 5-8, Honolulu, US, July 2006.

[4] T. Yanagawa, S. Aoki, T.Ohyama, "Human finger vein images are diverse and its patterns are useful for personal identification," Kyushu University $\mathrm{MHF}$ Preprint Series, Kyushu, Japan, pp. 1-7, 
2007.

[5] N. Miura, A. Nagasaka , T Miyatake, "Feature extraction of finger-vein patterns based on repeated line tracking and its application to personal identification," Machine Vision and Applications, vol. 15, pp. 194-203, July 2004.

[6] Z. Zhang, S. Ma, X Han. "Multiscale feature extraction of finger-vein patterns based on curvelet and local interconnection structure neural network," In Proceedings of 18th international conference on pattern recognition, pp. 145-148, Hong Kong, China, Aug. 2006.

[7] J D. Wu, C T. Liu. "Finger-vein pattern identification using principal component analysis and the neural network technique," Expert Systems with Application. vol. 35, no. 5, pp: 5423-5427, May 2011.

[8] E C. Lee, H W. Jung, D Y. Kim, "New finger biometric method using near infrared imaging," Sensors, vol. 11, no. 3, pp. 2319-2333, Feb. 2011.

[9] J F. Yang, X. Zhang, "Feature-level fusion of global and local features for finger-vein recognition," In Proceeding of the $10^{\text {th }}$ International Conference on Signal Processing. pp. 1702-1705, Beijing, China, Oct. 2010.

[10] F X. Guan, K J. Wang, H W. Mo, H. Ma, J Y. Li, "Research of finger vein recognition based on fusion of wavelet moment and horizontal and vertical 2DPCA," In Proceeding of 2nd International Congress on Image and Signal Processing, pp. 1-5, Tianjin, China, Oct. 2009.

[11] Y. Yang, G P. Yang, S B. Wang. "Finger vein recognition based on multi-instance," International Journal of Digital Content Technology and its Applications, vol. 6, no. 11, pp. 86-94, June, 2012.

[12] J F. Yang, M F. Yan, "An improved method for finger-vein image enhancement," In Proceeding of the 10th International Conference on Signal Processing, pp.
1706-1709, Beijing, China, Oct. 2010.

[13] J. Zhang, J F. Yang, "Finger-vein image enhancement based on combination of gray-level grouping and circular Gabor filter," in Proceeding of International Conference on Information, Electronic and Computer Science, pp. 1-4, Wuhan, China, Dec. 2009.

[14] J. Wang, Y. Tan, "Morphological image enhancement procedure design by using genetic programming," In Proceeding of the $13^{\text {th }}$ annual conference on Genetic and evolutionary computation, pp. 1435-1442, Dublin, Ireland, July 2011.

[15] J F. Yang, B. Zhang, "Scatting removal for finger-vein image enhancement," In Proceeding of International Conference on Hand-based Biometrics. pp. 1-5, Hong Kong, China, Nov. 2011.

[16] K K. Gupta and R. Gupta, "Feature adaptive wavelet shrinkage for image denoising," in The Proc. of International Conference on Signal Processing, Communications and Networking, pp. 81-85, Chennai, India, Feb. 2007.

[17] K. Zuiderveld, "Contrast limited adaptive histogram equalization," Graphics gems IV, Academic Press Professional, (pp. 474-485), 1994.

[18] L. Ma, Y H. Wang, T N. Tan, "Iris recognition based on multichannel Gabor filtering," In Proceeding of the International Conference on Asian Conference on Computer Vision, pp. 279-283, Melbourne Australia, Jan. 2002

[19] G. Carsten, "Curved-region-based ridge frequency estimation and curved Gabor filters for fingerprint image enhancement," IEEE Transaction on Image Processing. vol. 24, no. 4, pp. 2220-2227, April 2012

[20] M. Choi, S. Lee, S. Sin, K. Jank, M. Jung, "A Study of Vein Identification System using 2D-Garbor Filter for the Vessel USN Entrance/Exit Management,", The Journal of Korean Information and Communications 
Scociety, vo.1 32, no. 11, pp. 1190-1196, June 2007

[21] A K. Jain, S. Prabhakar, S. Pankanti, "Filterbank-based fingerprint matching," IEEE Transaction on Image Processing, vol. 9, no. 5, pp. 846-859, May 2000.

[22] A. Jain, K. Nandakumar, A. Ross, "Score normalization in multimodal biometric systems," Pattern Recognition, vol. 38, pp. 2270-2285, Dec. 2005.

[23] X C. Wang, J. Xu, Z. Li, "A new multimodal biometric identification method with small sample size based on iris and palmprint integration," JCIT: Journal of Convergence Information Technology, vol. 6, no. 5, pp. 51-60, May 2011.

[24] M X. He, S J. Horng, P Z. Fan, R S. Run, et al, "Performance evaluation of score level fusion in multimodal biometric systems", Pattern Recognition, vol. 43, pp. 1789-1800, May 2010.

[25] Park, K. Ryong, "Finger vein recognition by combing global and local feature base on SVM," Computing and Informatics, vol. 30, no. 2, pp. 295-309, April 2011.

[26] Yilong Yin, SDUMLA-HMT database, Jan. 2012, from http://mla.sdu.edu.cn/sdumla-hmt.html

[27] H V. Nguyen, L. Bai, "Cosine similarity metric learning for face verification," Lecture Notes in Computer Science, vol. 6493, pp. 709-720, 2010.

[28] I. T. Jolliffe, "Principal Component Analysis," Springer Series in Statistics, 2nd ed, Springer-Verlag New York, 2002.

[29] J F. Yang, Y H. Shi, J L. Yang, L H. Jiang, "A novel finger-vein recognition method with feature combination," The Proceeding of 16th IEEE International Conference on Image Processing, pp. 2709-2712, Cairo, Egypt, Nov. 2009.
Yu Lu

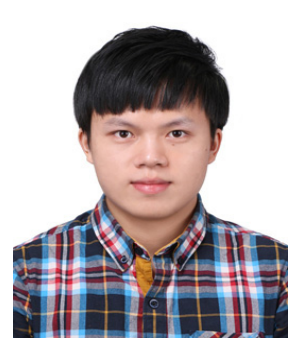

He received his B.S degree in Nanchang Institute of Technology, China in 2008. And in 2011, he received his M.S degree in Jiangxi University of Finance and Economics, China. Now he is a $\mathrm{Ph}$. D student with the major of Electronic Engineering in Chonbuk National University. Korea. His Research interests includes biometric feature recognition, computer vision and image processing.

\section{Sook Yoon}

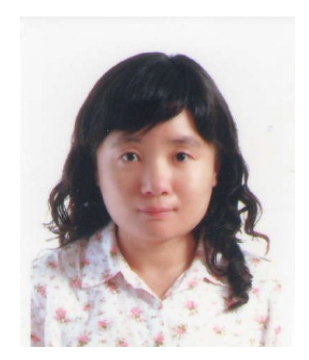

She received the B.S., M.S., and Ph.D. degrees in engineering from Chonbuk National University, Jeonbuk, Korea, in 1993, 1995, and 2003, respectively. Until June

2006, she conducted her postdoctoral research work in electrical engineering at the University of California, Berkeley. She is presently a associate professor at Department of Multimedia Engineering, Mokpo National University, Jeonnam, Korea. Her current research interests include image processing, pattern recognition, machine learning, and multimedia computing.

\section{Dong Sun Park}

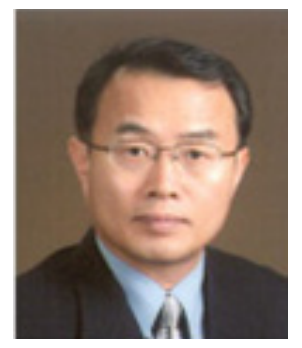

He received the B.S. degree from Korea University, Seoul, Korea, in 1979, and the M.S. and Ph.D. degrees from the University of Missouri, Columbia, in 1984 and 1990, respectively.

$\mathrm{He}$ is currently a Professor with the School of Electronic Engineering, Chonbuk National University, Jeonbuk, Korea. His current research interests include image processing, pattern recognition, computer vision, and artificial intelligence. 